\title{
A new era of systemic therapy for intermediate and advanced stage hepatocellular carcinoma
}

\author{
Masatoshi Kudo \\ Department of Gastroenterology and Hepatology, Kindai University Faculty of Medicine, Osaka, Japan \\ Correspondence to: Masatoshi Kudo, MD, PhD. Department of Gastroenterology and Hepatology, Kindai University Faculty of Medicine, Osaka, \\ Japan. Email: m-kudo@med.kindai.ac.jp. \\ Provenance and Peer Review: This article was commissioned by the editorial office, Hepatobiliary Surgery and Nutrition. The article did not undergo \\ external peer review.
}

Submitted Apr 10, 2020. Accepted for publication May 13, 2020.

doi: $10.21037 / \mathrm{hbsn}-20-420$

View this article at: http://dx.doi.org/10.21037/hbsn-20-420

Until recently, transarterial chemoembolization (TACE) was the only standard treatment for intermediate-stage hepatocellular carcinoma (HCC) according to the worldwide guidelines for the diagnosis and treatment of HCC $(1,2)$. However, TACE is not always effective in providing a survival benefit because of the extreme heterogeneity of intermediate-stage HCC regarding tumor size and number, and liver function (Child-Pugh A5-B9). In a review article, Bruix et al., the developer of the Barcelona Clinic Liver Cancer staging system, stated that TACE is not indicated for all cases of intermediate-stage HCC (3). Therefore, other treatments are required to cover these unmet needs, especially when the tumor burden is relatively large. To this end, various attempts have been made to subclassify intermediate-stage HCC into substages to develop more specific treatment strategies (4). Initial treatment with lenvatinib was recently proposed for HCCs beyond the up-to-seven criteria $(5,6)$ (Figure 1) because it showed high response rates in intermediate-stage HCC patients $(61 \%)(7)$ in the subanalysis of the REFLECT trial $(5,8)$. Patient outcomes are further improved by the subsequent use of selective TACE $(6,9)$. This novel treatment strategy may extend the survival of patients with high tumor burden for the following reasons: (I) it causes tumor necrosis and shrinkage; (II) tumor vessel normalization by lenvatinib increases the efficacy of subsequent TACE; and (III) it suppresses the increase of cytokines, such as vascular endothelial growth factor or angiopoietin 2, that follows TACE-induced hypoxia (6). The validity of this strategy was tested in the TACTICS trial, in which sorafenib was used as the initial therapy $(10,11)$. The results of a recent proof-ofconcept trial indicate that HCCs with high tumor burden treated with initial lenvatinib followed by subsequent selective TACE show a complete response, leading to a real cure after discontinuation of lenvatinib in some cases, suggesting that LEN-TACE sequential therapy may become the standard-of-care for patients who are unsuitable for TACE $(6,9)$ (Figure 1). In addition, curative therapy such as resection or ablation is feasible after downstaging by lenvatinib. Initial lenvatinib therapy followed by selective TACE can significantly improve patient prognosis and objective response rate (ORR) compared with the effects of TACE alone because of the direct tumor necrotizing effect of selective TACE $(6,12)$.

The IMbrave 150 Phase III trial tested the use of atezolizumab plus bevacizumab for the treatment of advanced-stage HCC patients. The results were published in New England fournal of Medicine in 2020 (13), leading to a change in the paradigm of HCC treatment. A total of 501 patients were randomized 2:1 to the atezolizumab plus bevacizumab group or sorafenib group. The endpoints of this trial were overall survival (OS) and progressionfree survival (PFS). The first interim analysis indicated that combination treatment with atezolizumab and bevacizumab resulted in significantly longer OS and PFS than those achieved with sorafenib alone. The median OS of patients receiving atezolizumab $(1,200 \mathrm{mg} / \mathrm{kg}$ iv q3w) in combination with bevacizumab $(15 \mathrm{mg} / \mathrm{kg} \mathrm{q} 3 \mathrm{w})$ was not estimable (NE), whereas the median OS for sorafenib was 13.2 months (95\% CI, 10.4-NE), with a hazard ratio 

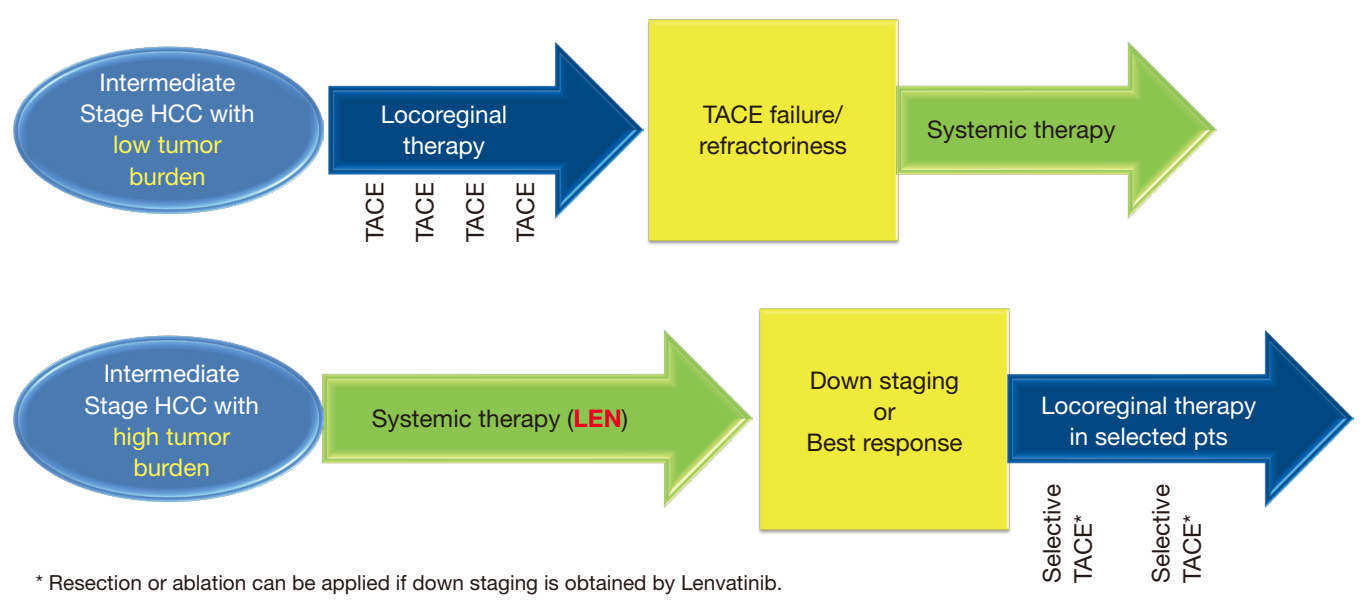

Figure 1 Changing concept for treatment strategy in intermediate-stage HCC with high tumor burden. HCC, hepatocellular carcinoma.

Systemic Therapy in HCC: 2020 and Beyond

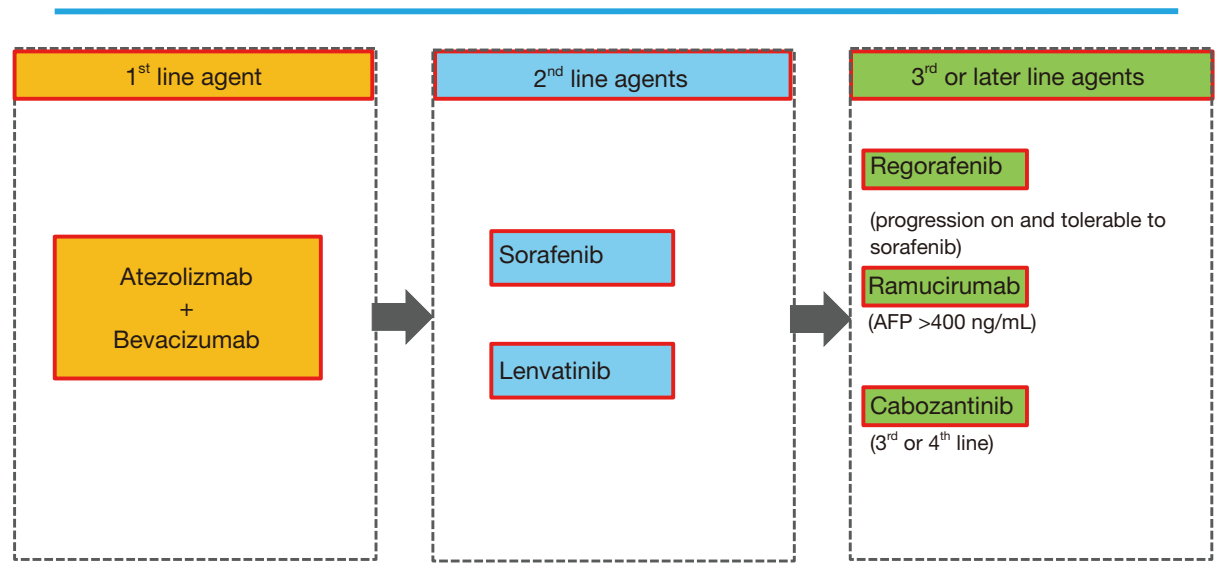

Figure 2 Systemic therapy in HCC: 2020 and beyond. HCC, hepatocellular carcinoma.

of 0.58 (95\% CI, 0.42-0.79, $\mathrm{P}=0.0006$ ). The median PFS and ORR in the group that received atezolizumab plus bevacizumab were 6.8 months (95\% CI, 5.7-8.3) and $27 \%$, compared with 4.3 months (95\% CI, 4.0-5.6) and $12 \%$ in the sorafenib group, respectively (PFS hazard ratio, 0.59; $95 \%$ CI, 0.47-0.76; $\mathrm{P}<0.0001$ for both cases). The patientreported outcome, i.e., the time to deterioration of quality of life (QOL), was more favorable in the atezolizumab plus bevacizumab group, showing a significantly prolonged QOL decline compared with that in the sorafenib group (12.1 vs. 3.6 months). Furthermore, combination treatment showed a better safety profile than sorafenib alone. These results indicate that atezolizumab plus bevacizumab will replace lenvatinib and sorafenib as first-line strategies after it is approved for clinical use, and that it may displace other drug therapies one level down (Figure 2).

Disease progression from the baseline is observed in approximately $20 \%$ of cases treated with atezolizumab in combination with bevacizumab. In addition, because both drugs are monoclonal antibodies, the resulting decline in liver function is expected to be less severe than that caused by tyrosine kinase inhibitors. Therefore, it is estimated that $80-90 \%$ of cases showing progressive disease with atezolizumab plus bevacizumab therapy will move to secondline and later line therapies. Therefore, even in patients with advanced-stage HCC, molecular targeted drugs such as lenvatinib and sorafenib, or later line drugs such as regorafenib, ramucirumab, and cabozantinib, will continue 
to play an important role, including after treatment with atezolizumab plus bevacizumab. In that sense, accurately identifying TACE-unsuitable patients with high tumor burden is critical. In addition, when possible, avoiding the overuse of TACE is important because it decreases hepatic functional reserve, which may lead to a missed chance to receive sequential systemic therapy.

In conclusion, the treatment paradigm for intermediateand advanced-stage HCC is drastically changing. The treatment paradigm in patients with intermediate-stage HCC is shifting markedly because of the emergence of a new treatment option, LEN-TACE sequential therapy. In advanced-stage HCC, combination immunotherapy with atezolizumab plus bevacizumab will become the first-line treatment replacing lenvatinib or sorafenib. Clinical trials of combination immunotherapy are ongoing and include all stages of HCC, from early-stage to advanced-stage $(14,15)$, such as the adjuvant setting after resection or ablation for early stage HCC or combination therapy with TACE for intermediate stage HCC. These changes in the treatment paradigm of HCC are expected to markedly improve the prognosis of patients.

\section{Acknowledgments}

Funding: None.

\section{Footnote}

Conflicts of Interest: The author has completed the ICMJE uniform disclosure form (available at http://dx.doi. org/10.21037/hbsn-20-420). Lectures: Eisai, Bayer, MSD, Eli Lilly; Grants: EA Pharma, Eisai, Gilead, Takeda, Otsuka, Taiho; Advisory Consulting: Eisai, Ono, MSD, BMS, Roche, AstraZeneca, Eli Lilly.

Ethical Statement: The author is accountable for all aspects of the work in ensuring that questions related to the accuracy or integrity of any part of the work are appropriately investigated and resolved.

Open Access Statement: This is an Open Access article distributed in accordance with the Creative Commons Attribution-NonCommercial-NoDerivs 4.0 International License (CC BY-NC-ND 4.0), which permits the noncommercial replication and distribution of the article with the strict proviso that no changes or edits are made and the original work is properly cited (including links to both the formal publication through the relevant DOI and the license). See: https://creativecommons.org/licenses/by-nc-nd/4.0/.

\section{References}

1. Marrero JA, Kulik LM, Sirlin CB, et al. Diagnosis, Staging, and Management of Hepatocellular Carcinoma: 2018 Practice Guidance by the American Association for the Study of Liver Diseases. Hepatology 2018;68:723-50.

2. EASL Clinical Practice Guidelines: Management of hepatocellular carcinoma. J Hepatol 2018;69:182-236.

3. Bruix J, Reig M, Sherman M. Evidence-Based Diagnosis, Staging, and Treatment of Patients With Hepatocellular Carcinoma. Gastroenterology 2016;150:835-53.

4. Kudo M, Arizumi T, Ueshima K, et al. Subclassification of BCLC B Stage Hepatocellular Carcinoma and Treatment Strategies: Proposal of Modified Bolondi's Subclassification (Kinki Criteria). Dig Dis 2015;33:751-8.

5. Kudo M. Extremely High Objective Response Rate of Lenvatinib: Its Clinical Relevance and Changing the Treatment Paradigm in Hepatocellular Carcinoma. Liver Cancer 2018;7:215-24.

6. Kudo M. A New Treatment Option for Intermediate-Stage Hepatocellular Carcinoma with High Tumor Burden: Initial Lenvatinib Therapy with Subsequent Selective TACE. Liver Cancer 2019;8:299-311.

7. Yamashita T, Kudo M, Ikeda K, et al. REFLECT-a phase 3 trial comparing efficacy and safety of lenvatinib to sorafenib for the treatment of unresectable hepatocellular carcinoma: an analysis of Japanese subset. J Gastroenterol 2020;55:113-22.

8. Kudo M, Finn RS, Qin S, et al. Lenvatinib versus sorafenib in first-line treatment of patients with unresectable hepatocellular carcinoma: a randomised phase 3 noninferiority trial. Lancet 2018;391:1163-73.

9. Kudo M, Ueshima K, Chan S, et al. Lenvatinib as an Initial Treatment in Patients with Intermediate-Stage Hepatocellular Carcinoma Beyond Up-To-Seven Criteria and Child-Pugh A Liver Function: A Proof-Of-Concept Study. Cancers (Basel) 2019;11.

10. Kudo M, Ueshima K, Ikeda M, et al. Randomised, multicentre prospective trial of transarterial chemoembolisation (TACE) plus sorafenib as compared with TACE alone in patients with hepatocellular carcinoma: TACTICS trial. Gut 2020;69:1492-501.

11. Kudo M. Proposal of Primary Endpoints for TACE Combination Trials with Systemic Therapy: Lessons Learned from 5 Negative Trials and the Positive 
TACTICS Trial. Liver Cancer 2018;7:225-34.

12. Kudo M. Lenvatinib may drastically change the treatment landscape of hepatocellular carcinoma. Liver Cancer 2018;7:1-19.

13. Finn RS, Qin S, Ikeda M, et al: Atezolizumab plus Bevacizumab in Unresectable Hepatocellular Carcinoma.

Cite this article as: Kudo M. A new era of systemic therapy for intermediate and advanced stage hepatocellular carcinoma. HepatoBiliary Surg Nutr 2020;9(4):530-533. doi: 10.21037/ hbsn-20-420
N Engl J Med 2020;382:1894-905.

14. Kudo M. Combination Cancer Immunotherapy in Hepatocellular Carcinoma. Liver Cancer 2018;7:20-7.

15. Kudo M. Immuno-Oncology Therapy for Hepatocellular Carcinoma: Current Status and Ongoing Trials. Liver Cancer 2019;8:221-38. 